\title{
A Study of Short Term Outcome of Management of Locally Advanced Breast Cancer
}

\author{
Dr. S. J. Bhosale ${ }^{1}$, Dr. Shruti C. Panicker ${ }^{2}$ \\ Professor Department of General Surgery \\ Resident, Department of General Surgery
}

\begin{abstract}
Introduction: Locally advanced breast cancer (LABC) continues to be a significant problem in the country and a common breast cancer presentation worldwide. LABC generally is defined as bulky primary chest wall tumours and/or extensive adenopathy. This includes patients with $\mathrm{T3}(>5 \mathrm{~cm})$ or T4 tumours (chest wall fixation or skin ulceration and/or satellitosis) and N2/N3 disease (matted axillary and/or internal mammary metastases). Objective: In this study, an attempt will be made to study the various risk factors like age, family history, parity, breast feeding, various modes of presentation of LABC and with special emphasis on locally advanced breast carcinoma and the multi-modality treatment approach (that includes neo-adjuvant chemotherapy, surgery and post-surgical radiotherapy, chemotherapy and hormone therapy). Material and Methods: This is a clinical prospective study.As per our protocol, patients were treated with neo- adjuvant chemotherapy followed by palliative mastectomy with axillary dissection, then chemotherapy, radiotherapy, and hormone therapy.Cases were followed up till the end of the study, the follow up included clinical examination for recurrence of the tumour at the operated site and recurrence in axilla.After completion of the study of the proven fifty cases of carcinoma breast the findings were analysed and compared with other studies. Results: Among the patients in the present study, 30\% patients received all five modalities of treatment (neo, surgery, chemotherapy, radiotherapy and hormonal therapy).42\% patients received four modalities of treatment (surgery, chemotherapy, radiotherapy, hormonal therapy). 68\% received only three modalities of treatment. This is attributed to poor patient compliance for neo adjuvant chemotherapy and post op radiation.Patients who received all the modalities of treatment and kept regular follow up were found to have no loco regional recurrence in the short term (18 months) of the study conducted.
\end{abstract}

Keywords: Locally advanced breast cancer, multi-modality treatment, risk factors

\section{Introduction}

One readily recognizes the impact of breast carcinoma in human societies, as this problem continues to increase exponentially, carcinoma breast is one of the most common carcinoma occurring in females and it is a devastating illness both physically and mentally. ${ }^{1}$

Breast cancer stands next to carcinoma of cervix as the most common cause of cancer deaths. ${ }^{2}$ In India there is an annual incidence of 75,000 cases per year. In developing countries like India women have 1 in 22 chance of developing carcinoma breast where as in USA the chance is 1 in 8 , the chance of getting carcinoma increases as age increases. ${ }^{3}$

Carcinoma breast is one of the most common carcinoma occurring in female .Breast cancer stands next to carcinoma of cervix as the most common cause of cancer deaths .In India there is annual incidence of 75,000 cases per year. Breast cancer causes 5,19,000 deaths in a year worldwide, about 9,00,000 women are diagnosed each year. Incidence of breast cancer is $0.26 / 1,00,000$ in males and $20.01 / 1,00,000$ in females. While mortality associated with breast cancer is 1.20/1,00,000 in males and 4.32/1,00,000 in females. Mortality rates from breast cancer have increased during the past 60 years in every country ${ }^{3}$.

Locally advanced breast cancer (LABC) continues to be a significant problem in the country and a common breast cancer presentation worldwide. LABC generally is defined as bulky primary chest wall tumours and/or extensive adenopathy. This includes patients with T3 $(>5 \mathrm{~cm})$ or T4 tumours (chest wall fixation or skin ulceration and/or satellitosis) and N2/N3 disease (matted axillary and/or internal mammary metastases) ${ }^{6,7}$. The extent to which LABC represents neglect and delayed diagnosis versus aggressive tumor biology is unclear.

Locally advanced breast cancer is subdivided into two major categories, in situ disease, mainly in the form of ductal carcinoma in situ (DCIS), and invasive cancer. Both are heterogeneous processes with very variable appearances, biology and clinical behavior.

These disparities partly reflect socioeconomic and health care access inequalities, but parallel variations in the incidence of breast cancer based on country of origin also suggest the existence of environmental and genetic factors.

It is most often observed that due to lack of knowledge and ignorance, patients of carcinoma breast clinically present in a late stage of the disease. The management of breast cancer requires a complex multidisciplinary approach involving surgeons, radiotherapists, medical oncologists, and pathologists.

In this study, an attempt will be made to study the various risk factors like age, family history, parity, breast feeding, various modes of presentation of LABC and with special emphasis on locally advanced breast carcinoma and the multi-modality treatment approach (that includes neoadjuvant chemotherapy, surgery and post-surgical radiotherapy, chemotherapy and hormone therapy). 


\section{Aims and Objectives}

- To study the short term outcome of locally advanced breast cancer

- To study various modalities of management OF LOCALLY ADVANCED BREAST CANCER.

\section{Methodology}

\section{Source of Data}

The material for this study was obtained from patients who presented to the surgical OPD of Krishna Institute If Medical Sciences and Hospital with locally advanced breast carcinoma from $1^{\text {st }}$ November 2013 to August 2015.

\section{Inclusion Criteria}

Patients with T3/T4 tumors (with chest wall fixity/ skin ulceration and/or satellitosis) and N2/N3 disease ( matted axillary/ internal mammary/ supraclavicular nodes on ipsilateral side.

\section{Exclusion Criteria}

- Early carcinoma breast with stage T1/T2, N0/N1

- Extensive metastasis at the time of presentation.

\section{Method of Collecting Data}

This is a clinical prospective study.As per our protocol, patients were treated with neo- adjuvant chemotherapy followed by palliative mastectomy with axillary dissection, then chemotherapy, radiotherapy, and hormone therapy.

Cases were followed up till the end of the study, the follow up included clinical examination for recurrence of the tumour at the operated site and recurrence in axilla.

After completion of the study of the proven fifty cases of carcinoma breast the findings were analysed and compared with other studies over a period of 18 months.

\section{Results}

The study was conducted in Krishna institute is medical sciences and research centre, Karad, for a period of 18 months from Nov 2013 to June 2015. Fifty patients of locally advanced breast cancer were included in this study, and were studied in detail.The incidence of locally advanced breast cancer was highest in patients between 40-50 years of age , with a mean age of 48.90 with a SD of 9.51 years.57.1\% patients presented to the hospital within 6 months of onset of symptoms. But early presentation does not mean early disease. Most of the patients included in the study belonged to the lower socioeconomic group. This can be concluded by the fact that this is a teaching hospital and all the patients received treatment that was included under the government run scheme for the weaker section of the society. About $60 \%$ of the patients included in the study were in the postmenopausal group. The incidence of breast carcinoma increases with longer period of reproductive life. In this study, $80 \%$ of the patients had fertile period of $25-34$ year.62\% patients had lump in the left breast and 14.2\% patients had the outer upper quadrant involved. Multiple quadrants involvement was the commonest presentation in this study. Lump in the breast was the commonest presentation $(60 \%) 24 \%$ patients presented with peud' orange, $42 \%$ with nipple retraction, $40 \%$ with ulceration.10\% patients presented with fixity of the breast tissue to the chest wall. In the present study all the patients presented with a lump of size $5 \mathrm{~cm}$ or more. (2\%) had $5 \mathrm{~cm}$, (24\%) had $6 \mathrm{~cm}$, (36\%) had $7 \mathrm{~cm}$ and remaining had more than $7 \mathrm{~cm} .86 \%$ patients presented with axillary lymph node involvement clinically, $16 \%$ had supraclavicular involvement of the ipsilateral side. Out of the fifty patients included in the study $36 \%$ (18) were stage III a and 64\% (32) were stage IIIb. The commonest type of carcinoma was infiltrating duct carcinoma (82\%).The accuracy of FNAC correlating with histopathology was found to be $100 \%$ in this series. In the present study, $46 \%$ patients had ER/PR positive status and $10 \%$ of patients had ER/PR negative status. Treatment plan followed for the patients included in this study was multidisciplinary. In this series, we were able to follow up 50 patients for a period of 6 months to 18 months. The remaining patients (6) did not turn up for regular follow up. Patients were examined, investigated and treated during follow up. In the present study $46 \%$ patients underwent neo adjuvant chemotherapy, all patients underwent surgery,92\% patients underwent post- surgery chemotherapy,82\% underwent radiotherapy, and 76\% underwent hormone therapy. Among the patients in the present study, 30\% patients received all five modalities of treatment (neo, surgery, chemotherapy, radiotherapy and hormo $42 \%$ patients received four modalities of treatment (surgery, chemotherapy, radiotherapy, hormonal therapy),68\% received only three modalities of treatment. This is attributed to poor patient compliance for neo adjuvant chemotherapy and post op radiation. In the present study no patients developed recurrence of disease or new tumor, 2 patients developed distant brain and bone metastasis .1 patients expired during the course of the disease. Patients who received all the modalities of treatment and kept regular follow up were found to have no loco regional recurrence in the short term (18 months) of the study conducted.

\begin{tabular}{|c|c|c|}
\hline Age in years & No. of cases & Percentage (\%) \\
\hline$<30$ & 1 & $2 \%$ \\
\hline $30-40$ & 9 & $18 \%$ \\
\hline $40-50$ & 22 & $44 \%$ \\
\hline $50-60$ & 11 & $22 \%$ \\
\hline $60-70$ & 7 & $14 \%$ \\
\hline Total & 50 & $100 \%$ \\
\hline Mean \pm SD & 48.90 years \pm 9.51 years \\
\hline Range & \multicolumn{2}{|c|}{27 years to 67 years } \\
\hline
\end{tabular}

Figure 1: Age distribution 


\section{International Journal of Science and Research (IJSR) \\ ISSN (Online): 2319-7064}

Index Copernicus Value (2013): 6.14 | Impact Factor (2015): 6.391

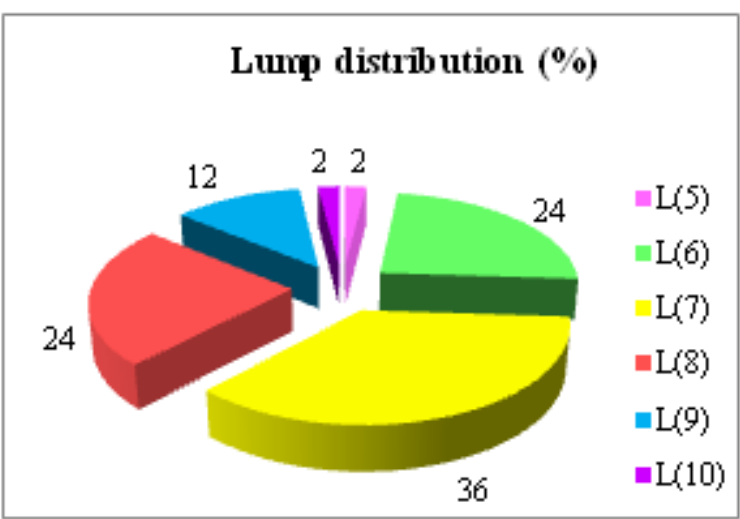

Figure 2: Lumpdistribution

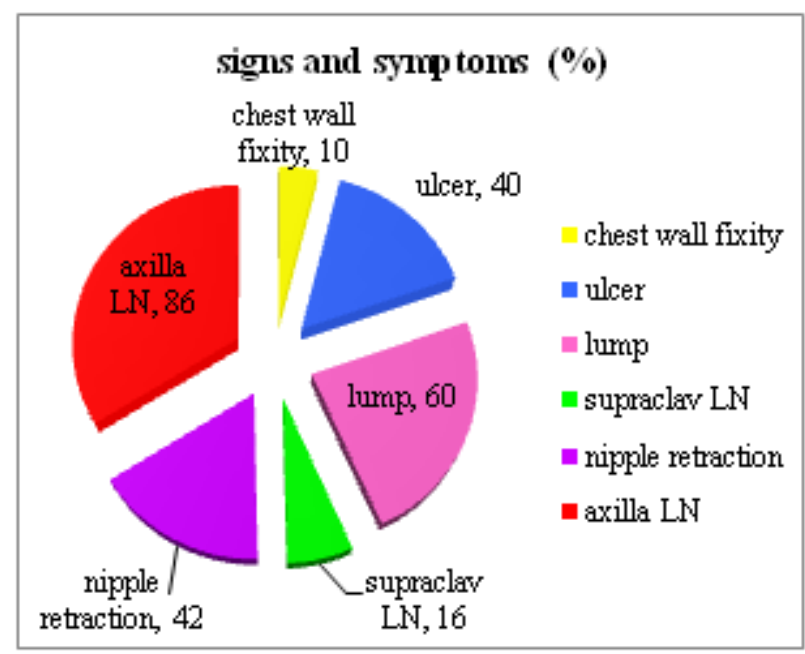

Figure 3: Signs and symptoms

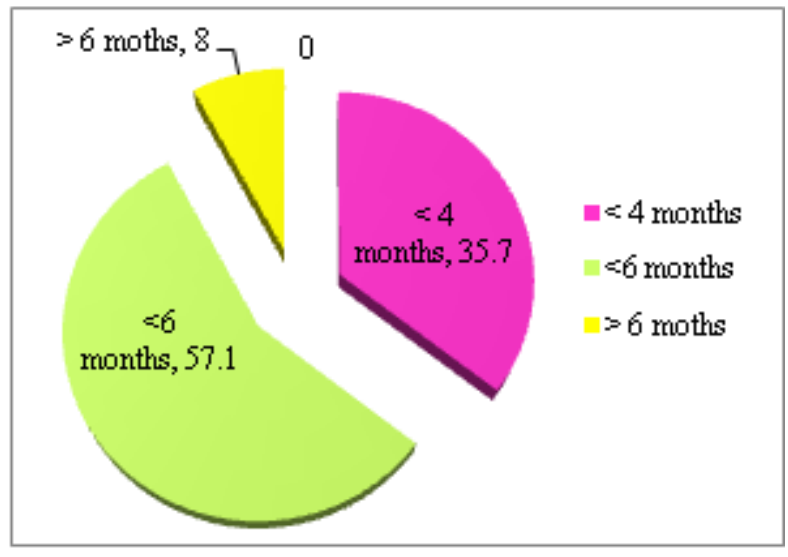

Figure 4: Duration of symptoms

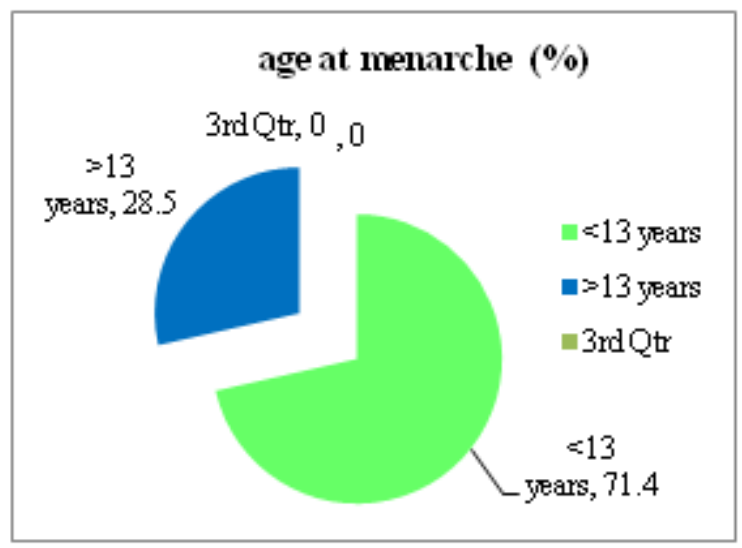

Figure 5: Age at menarche

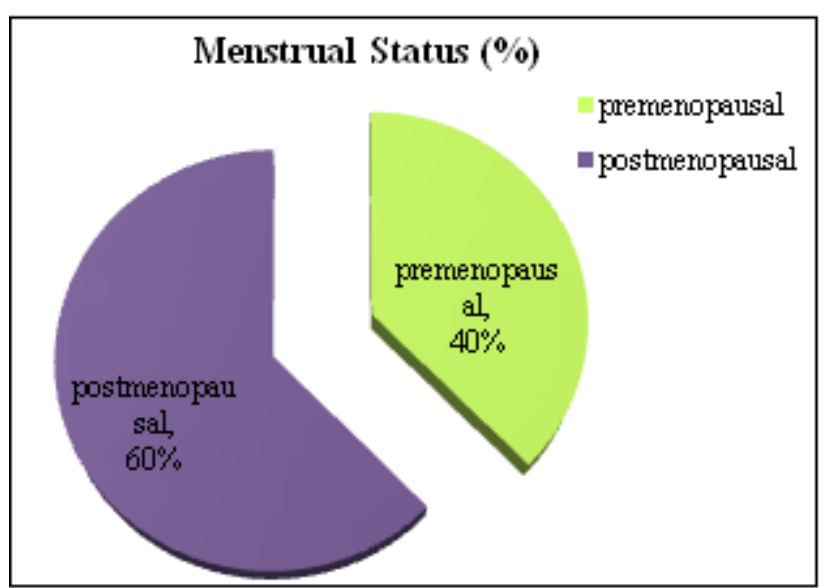

Figure 6: Menstrual status

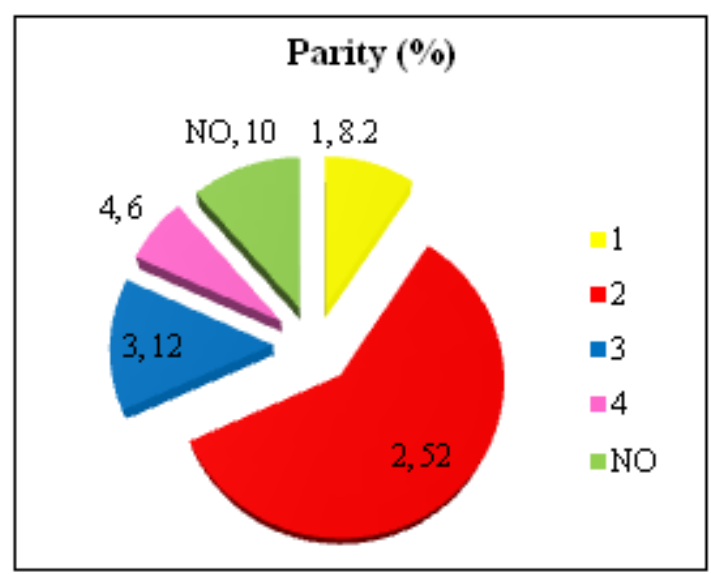

Figure 7: Parity

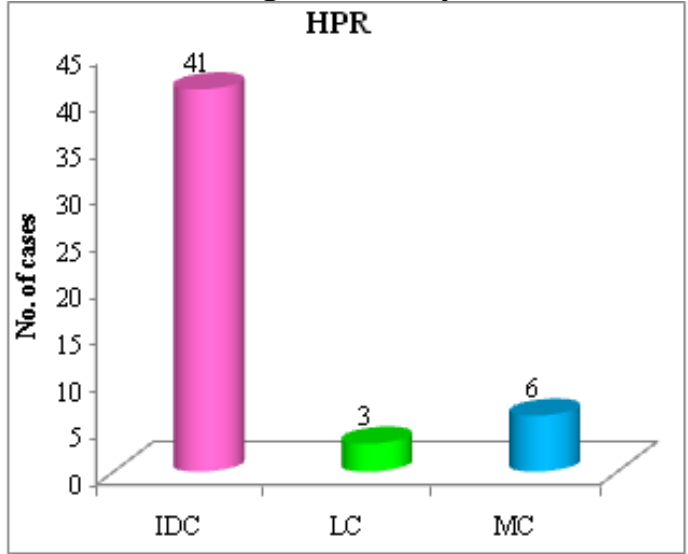

Figure 8: HPR

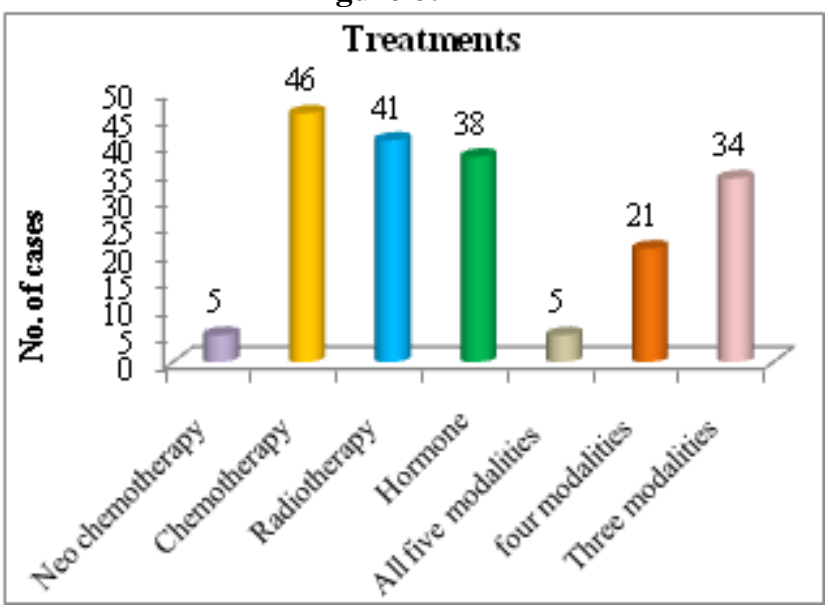

Figure 9: Treatment modalities

Volume 5 Issue 4, April 2016

www.ijsr.net 


\section{International Journal of Science and Research (IJSR) \\ ISSN (Online): 2319-7064}

Index Copernicus Value (2013): 6.14 | Impact Factor (2015): 6.391

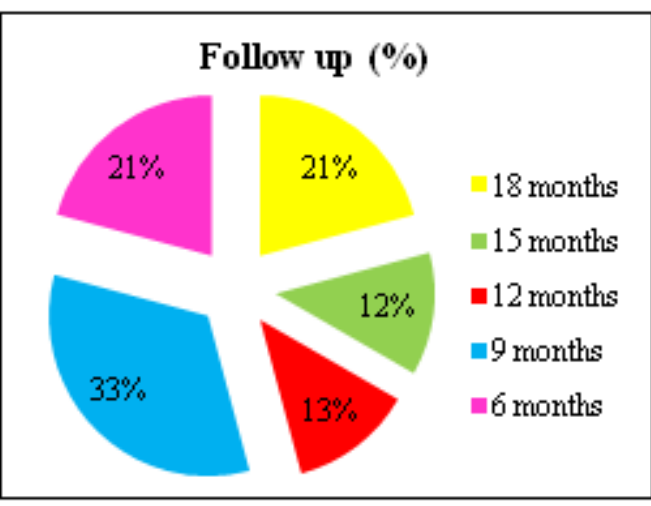

Figure 10: Follow up

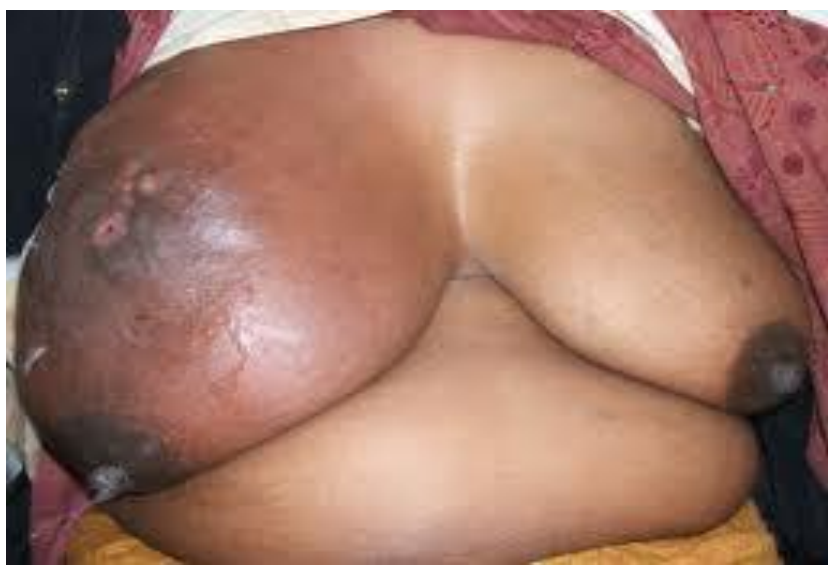

Figure 11: Locally Advanced Breast carcinoma

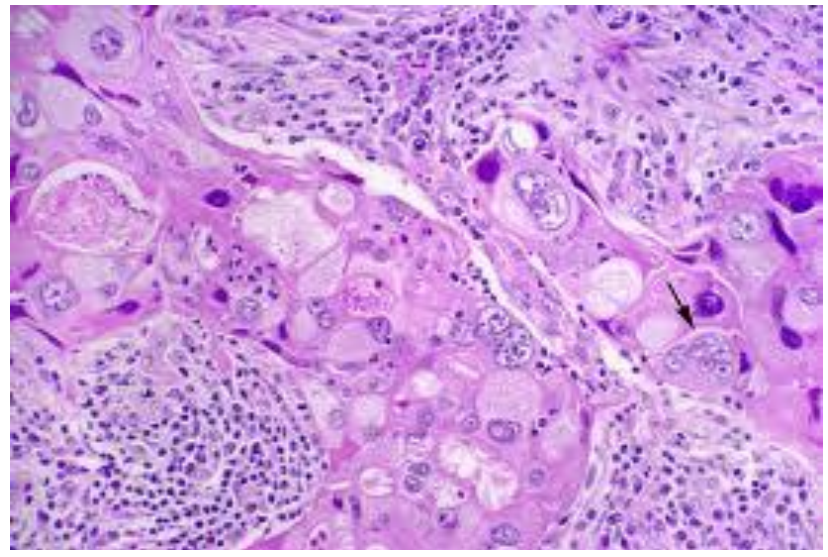

Figure 12: Infiltrating Duct Carcinoma

\section{Conclusion}

In the present series of fifty cases of locally advanced carcinoma of breast, the presentation of the patients was majority being between $40-50$ years of age group. Majority of patients belong to low socioeconomic strata of society, and most presenting at an advanced stage of the disease mainly due to lack of awareness about the seriousness of the disease and fear about the disease. Age at diagnosis remains an important factor for prognostication and treatment decisions in patients with breast cancer. Although, breast cancer in women below 40 years of age constitutes a small proportion of the total incidence, it has a significant burden on women and society.
Incidence rates and cumulative risk rates in women below 40 years vary little between populations, but generally remain low and do not justify screening in average risk women. Risk factors in breast cancer do not necessarily have the same effect in young and older patients. Breast cancer in young women is associated with a poorer outcome, partly because of over-representation of more aggressive subtypes, such as triple negative or HER2-positive breast cancer. In addition, they are more likely to present at an advanced stage or have a delayed diagnosis because of a low index of suspicion by the patient and the primary physician. These factors predispose to more loco-regional recurrences and distant metastases which contribute to the poorer outcome of young women with breast cancer. Locally advanced breast cancer remains a difficult clinical problem due to the high rate of relapse and low rate of overall survival. The range of disease encompassed by the term locally advanced is wide and includes patients with large, primary tumours to patients with rapidly progressing inflammatory carcinomas, who have very different prognosis. Thus, treatment decisions must be tailored to the individual patient. Of critical importance in all cases of locally advanced breast tumors is coordination of care among medical oncology, surgical oncology, and radiation oncology, as the vast majority of patients need chemotherapy, surgery, and radiotherapy. Overall outcome and local control rates have improved markedly with multimodal therapy, including neoadjuvant chemotherapy plus surgery and loco regional radiation. Additional postoperative systemic treatments are determined by primary tumor molecular markers.

\section{References}

[1] Copeland I, Gradisher, Introduction: The breast comprehensive management of benign\& malignant disease vol.1; chap1; WB Saunders; 2001

[2] Seidman $\mathrm{H}$ : probability of developing \& dying of cancer, cancer; 1985-134

[3] Http.// www. indiatimes.com/ women articles show/223923.cms

[4] 4.PeterJ.Morris, William c. Wood.Oxfords:Text book of surgery. $2^{\text {nd }}$ edition,Oxford University Press,Vol. 2: $1204 \mathrm{pp}$

[5] Frederick B wagner Jr, Samuel w. Beenken, Sr Kirby. I Bland: "History of Breast Cancer" The Breast comprehensive management of benign \& malignant disease: $3^{\text {rd }}$ edition , vol.1; chap1; WB Saunders; 2001

[6] GAG Decker, DJ Duphesis, Lee McGregors synopsis of surgical anatomy, $12^{\text {th }}$ Ed, KM Vargeepage $161-171$,

[7] McMinn, Last's Anatomy, $8^{\text {th }}$ ed.ELBS / Churchill Livingstone; 1998 8. Grey's anatomy, $39^{\text {th }}$ ed. ELSEVIER / Churchill Livingstone; 2004 